ISAHP 2001, Berne, Switzerland, August 2-4, 2001

\title{
NEW CONSISTENCY INDICES USING THE CARDINAL RELATIONSHIP IN PAIR-WISE COMPARISON MATRIX
}

\author{
Masanori Ozawa \\ Faculty of Science and Technology, Keio University \\ 3-14-1, Hiyoshi, Kohoku-ku, Yokohama 223-8522, Japan \\ ozawa@ae.keio.ac. \\ Yutaka Kato \\ College of Engineering, Hosei University \\ 3-7-2, Kjino-cho, Koganei, Tokyo 184-8584, Japan \\ kato@k.hosei.ac.jp
}

Keywords: consistency index, cardinal relationship, entropy function, simulation

Summary: In this study, we consider some consistency indices for a pair-wise comparison matrix. These indices derive from measuring the consistency gaps in the comparison matrix from a idempotent matrix. The consistency of each pair-wise comparison is defined by a cardinal relationship, then this cardinal relationship can be extended to consistency relationships in the comparison matrix. Some consistency indices are shown that those distance functions are a type of norms or a entropy function. By simulations, the idempotent relationship shows more sensitive than the residual relationship for consistency.

\section{Consistency of pair-wise comparison}

There are many types of the consistency of pair-wise comparison matrix, which are defined by a distance between the pair-wise comparison matrix and the model of AHP. Because the distance functions can be generated as matrix norms of the difference between matrices. Basically the consistency is introduced for each element of the comparison matrix as a cardinal relationship. Then the consistency of each element is defined by follows.

$$
a_{i j}=a_{i k} \cdot a_{k j}, \quad \text { for } k=1,2, \cdots, n
$$

In this definition, the element $a_{i j}$ must be satisfied above equation for all $i$ and $j$, but it is not required to satisfy the equation for all $k$. Then there is the next theorem for the consistency of comparison matrix.

\section{Theorem 1.}

Let $a_{i j}$ be a element of pair-wise comparison matrix of AHP, then the following statements are equivalent.

a) Cardinal relationship. For all $i$ and $j$,

$$
a_{i j}=a_{i k} \cdot a_{k j} \text {, for } k=1,2, \cdots, n .
$$

b) Idempotent relationship. The each element is satisfying,

$$
a_{i j}=\left\{\begin{array}{cc}
\frac{1}{n} \sum_{k=1}^{n} a_{i k} \cdot a_{k j}, & \text { for } i \neq j \\
1, & \text { for } i=j
\end{array} .\right.
$$

c) Residual relationship. There are positive numbers $w_{k} \geq 0, k=1,2, \cdots, n$ and the numbers are satisfied

$$
a_{i j}=w_{i} / w_{j}, \text { for } i, j=1,2, \cdots, n \text {. }
$$




\section{New consistency indices}

Now we can use the results of the theorem to evaluate the consistency of pair-wise comparison matrix. A consistency index can be formulated that is a difference between the terms of the equation in above theorem. Therefore we can make many consistency indices to use above theorem.

But in this time the condition (c) is mainly used to measure the consistency. For example, a consistency index is made by the condition (c) in the theorem and the maximum norm for measuring the differences in the equation (3).

$$
\left\|\left(a_{i j} w_{j} / w_{i}\right)\right\|_{\infty}=\max _{i=1, . ., n} \sum_{i=1}^{n}\left|a_{i j} w_{j} / w_{i}\right|
$$

In this case, if the estimated weights are the elements of the eigen vector of the comparison matrix, the value of the function is the consistency index by the eigen value. Conversely the weights which are to minimize this function are the elements of the eigen vector corresponding to the maximal eigen value.

\section{Simulation}

Now we generate comparison matrices to research characteristics of the consistency indices from the theorem. Then we calculate the consistency indices of the generated comparison matrices which are included a random error in each element. The plot curves in Figure 1. and 2 show the average of the indices which norm is the euclid norm. The generated comparison matrices in Figure 1. are included the $\log$ normal errors, which standard derivation is the horizontal axis in Figure 1. The errors in Figure 2. are generated by a kind of the normal distribution.

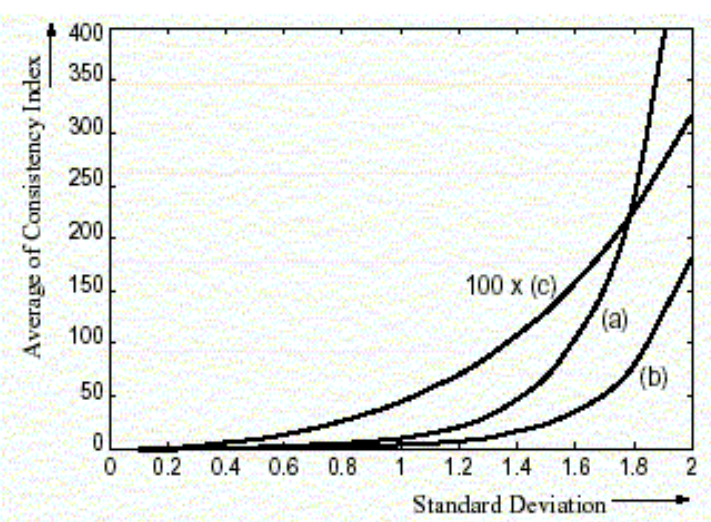

Figure 1: The average of consistency indices (the log normal distribution).

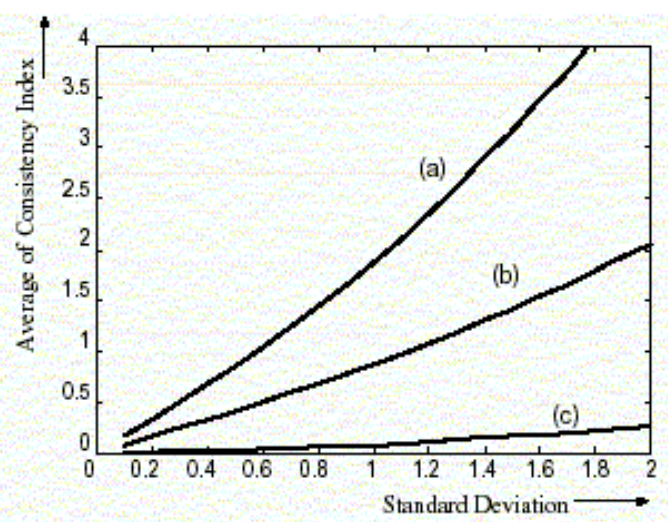

Figure 2: The average of consistency indices (a kind of the normal distribution).

\section{References}

[1] Saaty,T.L. "Eigenvector and logarithmic least squares", European Journal of OR, Vol.48, pp.156$160,1990$.

[2] Kato,Y., Ozawa,M. "The characteristics of the consistency function of the general mean method", pp.77-82, Proceedings of ISAHP'99, 1999. 